\title{
Modeling of multi-band drift in nanowires using a full band Monte Carlo simulation
}

Raghuraj Hathwar, Marco Saraniti, and Stephen M. Goodnick

Citation: Journal of Applied Physics 120, 044307 (2016); doi: 10.1063/1.4959881

View online: http://dx.doi.org/10.1063/1.4959881

View Table of Contents: http://aip.scitation.org/toc/jap/120/4

Published by the American Institute of Physics

\section{AlP | Journal of AlPplied Physics}

Save your money for your research.

It's now FREE to publish with us no page, color or publication charges apply.
Publish your research in the

to claim your place in applied

physics history. 


\title{
Modeling of multi-band drift in nanowires using a full band Monte Carlo simulation
}

\author{
Raghuraj Hathwar, Marco Saraniti, and Stephen M. Goodnick \\ School of Electrical, Computer and Energy Engineering, Arizona State University, Tempe, \\ Arizona 85287-8806, USA
}

(Received 4 April 2016; accepted 15 July 2016; published online 29 July 2016)

\begin{abstract}
We report on a new numerical approach for multi-band drift within the context of full band Monte Carlo (FBMC) simulation and apply this to $\mathrm{Si}$ and InAs nanowires. The approach is based on the solution of the Krieger and Iafrate (KI) equations [J. B. Krieger and G. J. Iafrate, Phys. Rev. B 33, 5494 (1986)], which gives the probability of carriers undergoing interband transitions subject to an applied electric field. The KI equations are based on the solution of the time-dependent Schrödinger equation, and previous solutions of these equations have used Runge-Kutta (RK) methods to numerically solve the KI equations. This approach made the solution of the KI equations numerically expensive and was therefore only applied to a small part of the Brillouin zone (BZ). Here we discuss an alternate approach to the solution of the KI equations using the Magnus expansion (also known as "exponential perturbation theory"). This method is more accurate than the RK method as the solution lies on the exponential map and shares important qualitative properties with the exact solution such as the preservation of the unitary character of the time evolution operator. The solution of the KI equations is then incorporated through a modified FBMC freeflight drift routine and applied throughout the nanowire BZ. The importance of the multi-band drift model is then demonstrated for the case of Si and InAs nanowires by simulating a uniform field FBMC and analyzing the average carrier energies and carrier populations under high electric fields. Numerical simulations show that the average energy of the carriers under high electric field is significantly higher when multi-band drift is taken into consideration, due to the interband transitions allowing carriers to achieve higher energies. Published by AIP Publishing.

[http://dx.doi.org/10.1063/1.4959881]
\end{abstract}

\section{INTRODUCTION}

The full band Monte Carlo method (FBMC) has been used to simulate high field transport in a variety of materials. It is a statistical method used to solve the Boltzmann transport equation by including the full band structure and scattering processes. The traditional method requires the calculation and storage of the band structure of the material on the full Brillouin zone (BZ). This is done through various methods such as the $\mathrm{k} \bullet \mathrm{p}$ method, ${ }^{1}$ the Empirical Pseudopotential method (EPM), ${ }^{2}$ the empirical Tight Binding method (ETB) ${ }^{3}$ etc. The calculation of the scattering rate is usually done using Fermi's golden rule for every initial state $\mathbf{k}$ to final state $\mathbf{k}^{\prime}$. The acceleration of carriers in the presence of an electric field is accomplished using the free-flight drift routine detailed in Ref. 4, which involves moving all the carriers according to the Bloch acceleration theorem for a time $t$ and then scattering the carriers according to their rates previously calculated at the end of the so-called free flight.

The free-flight drift routine is a single band model in which the carriers are assumed to remain in the same band during the drift. This model does not work well at band crossings or when the energy separation between the bands is small. At band crossings, it is difficult to maintain the carrier's band identity because the bands are sorted in energy after being calculated in an eigenvalue solver and there exists no unique way to sort the bands in energy. Therefore, when a carrier reaches a band crossing, for example, it is unclear which band it should follow after the crossing. ${ }^{5}$ An electric field can induce interband transitions ${ }^{6}$ when the energy separation between the bands are small, and the electric field is sufficiently large. This effect becomes prominent in materials such as nanowires where the energy spacing between the bands is small and the bands intercross and anticross frequently across the Brillouin Zone (BZ). The utility of the multi-band drift model is to replace the traditional free-flight drift routine in full band Monte Carlo models. After a carrier undergoes a drift for a particular duration of time, there is a finite possibility of it undergoing a transfer to the other bands. Various approaches have been proposed to incorporate carrier transitions between different bands such as the overlap test ${ }^{7}$ and the velocity continuity method. ${ }^{8}$ The overlap test calculates the overlap integral between the wave function at $k_{i}$ of a particular band index $\mathrm{j}$, to all the bands at $k_{i+1}$. The band index that has the largest overlap is assumed to have the band index $\mathrm{j}$ and the carrier is drifted to that band index as it moves from $k_{i}$ to $k_{i+1}$. If the $\mathrm{k}$-space gridding is very coarse, deciding which band has the largest overlap could be ambiguous. The velocity continuity method calculates the velocity $v_{i}$ at $k_{i}$ and tries to find a close match with the velocity $v_{i+1}$ at $k_{i+1}$ with all the available band indices. The band index with the closest velocity match is then 
assumed to be the correct band index and the carrier is drifted to that band index. Both these methods have drawbacks that the transition rate does not depend on the electric field and that it is sensitive to the mesh size in k-space.

A more rigorous approach to the problem was provided by Krieger and Iafrate (KI), who developed a set of equations which give the transition probability of a carrier under an electric field as a function of time. ${ }^{9}$ These equations are a series of complex partial differential equations, which were previously solved using the 4th order Runge-Kutta method (RK4). ${ }^{7,8,10}$ Since this method is computationally expensive, the KI equations were previously only solved on a small part of the BZ in bulk materials, where band crossings were expected to result in interband tunneling at high electric fields. In nanowires, the band crossings and interband tunneling can occur throughout the BZ due to the dense nature and mixing of the bands. Therefore, a fast and full BZ wide solution of the KI equations is necessary to properly account for interband tunneling in nanowires, as well as providing a more computationally efficient algorithm for treating interband tunneling in bulk materials as well.

We present numerical calculations of the multi-band transport and interband tunneling process in nanowires. A new method of solving the KI equations is also presented which greatly improves the accuracy and speed of the simulation. Section II discusses the KI equations, a new method to solve them, the methods numerical advantages over the RK4 method and its implementation in a FBMC routine. In Section III, we then discuss the results of the method applied to $\mathrm{Si}$ and InAs nanowires, followed by conclusions.

\section{MODEL DESCRIPTION}

\section{A. Krieger and lafrate equations}

The Krieger and Iafrate (KI) equations provides the solution of the time-dependent Schrodinger equation for Bloch waves under an electric field described by ${ }^{9}$

$$
H \psi(\mathbf{r}, t)=\left[\frac{[\mathbf{p}-(e / c) \mathbf{A}]^{2}}{2 m}+v(\mathbf{r})\right] \psi=i \hbar \frac{\partial \psi}{\partial t},
$$

where

$$
\mathbf{A}=-c \int_{0}^{t} F\left(t^{\prime}\right) d t^{\prime}
$$

and $v(\mathbf{r})$ is the crystal periodic potential, $c$ is the speed of light, and $F(t)$ is the time varying electric field. Substituting

$$
\psi(\mathbf{r}, t)=\sum_{n} C_{n}(t) \phi_{n}^{\prime}(\mathbf{r}, t)
$$

into Eq. (1), where $\phi_{n}^{\prime}(\mathbf{r}, t)$ is the solution to the eigenvalue problem

$$
\left[\frac{[\mathbf{p}-(e / c) \mathbf{A}]^{2}}{2 m}+v(\mathbf{r})\right] \phi_{n}^{\prime}(\mathbf{r}, t)=\varepsilon_{n}(t) \phi_{n}^{\prime}(\mathbf{r}, t),
$$

where $\varepsilon_{n}(t)$ are the eigenvalue solutions, we get the KI equations

$$
i \hbar \frac{\partial C_{n}(t)}{\partial t}=\varepsilon_{n}(t) \cdot C_{n}(t)+e F(t) \sum_{n^{\prime}} X_{n, n^{\prime}}(t) \cdot C_{n^{\prime}}(t),
$$

where $F(t)$ is the time dependent electric field, $-e$ is the electronic charge, $\varepsilon_{n}(t)$ is the eigenvalue of the nth band with wave vector $k(t)$, and $C_{n}(t)$ are time dependent coefficients and the $\mathrm{X}$ matrices given by

$$
X_{n, n^{\prime}}(k(t))=-i \int_{\Omega} u_{n, k(t)}^{*}(x) \cdot \nabla_{k} u_{n^{\prime}, k(t)}(x) \cdot d^{3} x,
$$

where $\Omega$ is the volume of the primitive cell, and $u_{n, k(t)}$ is the Bloch function for band $n$ for wave vector $k(t)$. The wave vector $k(t)$ is determined from the Bloch acceleration theorem

$$
\hbar \frac{\partial k(t)}{\partial t}=-e F(t)
$$

where $e$ is the charge of the carrier.

\section{B. Solution of the Krieger and lafrate equations}

Previous methods to solve the Krieger and Iafrate equations have used the Runge-Kutta method of the 4th order (RK4) to solve Eq. (5). ${ }^{7,8,10}$ This method required solving the coupled complex partial differential equations for every possible initial condition ( $n$ possible conditions if there are $n$ bands). This is numerically expensive as the time step required to solve the KI equations using the RK4 method can be very small $\left(\sim 10^{-18} \mathrm{~s}\right.$ to $\left.\sim 10^{-19} \mathrm{~s}\right)$. This time step becomes more problematic at higher electric fields as the time steps need to be readjusted to be progressively smaller according to the value of the electric field. Solutions to the KI equations are assumed to be correct if $\sum_{n}\left|C_{n}(t)\right|^{2} \approx 1$. The probability coefficients rapidly diverge if the time step is too large, so one has to continuously check the sum and recalculate the coefficients with a smaller time step if it is too large.

A more elegant solution can be obtained by using the Magnus expansion. ${ }^{11}$ Writing Eq. (5) in matrix notation we get

$$
\frac{\partial C(t)}{\partial t}=C(t) A(t)
$$

where $C(t)$ is an $n \times 1$ matrix and $A(t)$ is an $n \times n$ matrix, where $n$ is the number of bands and each element is given by

$$
A_{n n^{\prime}}(t)= \begin{cases}\frac{\varepsilon_{n}(t)}{i \hbar}-\frac{e F(t)}{i \hbar} X_{n n}(t) & n=n^{\prime} \\ -\frac{e F(t)}{i \hbar} X_{n n^{\prime}}(t) & n \neq n^{\prime} .\end{cases}
$$

Due to the nature of the problem, k-space and time are synonymous and related to each other by Eq. (7). It is easier to operate over k-space rather than time and therefore converting from $d t$ to $d k$, using Eq. (7), Eqs. (8) and (9) can be written as

$$
\frac{\partial C(k)}{\partial k}=C(k) A(k)
$$


where

$$
A_{n n^{\prime}}(k)= \begin{cases}-\frac{i \varepsilon_{n}(k)}{e F(k)}+i X_{n n}(k) & n=n^{\prime} \\ i X_{n n^{\prime}}(k) & n \neq n^{\prime} .\end{cases}
$$

Applying the Magnus expansion to Eq. (10), we get

$$
C\left(k_{f}\right)=\exp \left[\Omega\left(k_{f}\right)\right] C\left(k_{i}\right),
$$

where

$$
\Omega\left(k_{f}\right)=\sum_{j=1}^{\infty} \Omega_{j}\left(k_{f}\right) .
$$

The first two terms of the infinite series expansion are given by $^{9}$

$$
\begin{gathered}
\Omega_{1}\left(k_{f}\right)=\int_{k_{i}}^{k_{f}} A\left(k_{1}\right) d k_{1}, \\
\Omega_{2}\left(k_{f}\right)=\frac{1}{2} \int_{k_{i}}^{k_{f}} \int_{k_{i}}^{k_{1}}\left[A\left(k_{1}\right), A\left(k_{2}\right)\right] d k_{2} d k_{1},
\end{gathered}
$$

where $k_{i}$ is the value of the wave vector when $t=0$ and $k_{f}$ is the wave vector at $t=t_{1}$, and $\left[A\left(k_{1}\right), A\left(k_{2}\right)\right]=A\left(k_{1}\right) A\left(k_{2}\right)$ $-A\left(k_{2}\right) A\left(k_{1}\right)$ is the commutator operator. A recursive procedure to generate the 2 nd and higher terms in the Magnus expansion is given by ${ }^{12}$

$$
\Omega_{n}\left(k_{f}\right)=\sum_{j=1}^{n-1} \frac{B_{j}}{j !} \int_{k_{i}}^{k_{f}} S_{n}^{j}(k) d k,
$$

where

$$
\begin{aligned}
S_{n}^{j}(k) & =\sum_{m=1}^{n-j}\left[\Omega_{m}(k), S_{n-m}^{j-1}(k)\right] \\
S_{n}^{1}(k) & =\left[\Omega_{n-1}(k), A(k)\right],
\end{aligned}
$$

where $B_{j}$ are the Bernoulli numbers. The main advantage of the Magnus solution is that very often, the truncated series still has important qualitative properties of the exact solution. ${ }^{13}$ For example, regardless of the truncation of the Magnus series, $\sum_{n}\left|C_{n}(k)\right|^{2}$ is always equal to 1.0 due to the unitary nature of the solution. In fact, this is a property shared by all exponential perturbation methods such as the Fer method and the Wilcox method. ${ }^{14,15}$ This is not true in the case of RK4 methods or other perturbation methods like the Dyson series method.

Solving Eq. (12) requires the calculation of the exponential of a matrix. The calculation of the matrix exponential is usually numerically expensive for large matrices and scales as the cube of the matrix size, but in this case, the size of the matrix is the number of bands, which even in the case of nanowires, is at most 100 . Also, once the matrix is computed, the final probabilities can be calculated by a simple matrix multiplication with the initial conditions; thus, this approach is numerically advantageous over the RK method, in which the KI equations are simply recalculated for every possible initial condition.
To calculate the X matrices defined in Eq. (6), the calculation of the $\mathrm{k}$-space derivative of the Bloch function $u_{n, k(t)}$ is required. Two methods have been used to calculate the derivative, Rayleigh-Schrodinger perturbation theory (RSPT) ${ }^{16}$ and the finite difference method. Rayleigh-Schrodinger perturbation theory (RSPT) states that

$$
\nabla_{k} u_{n}(k)=\sum_{n^{\prime}, n^{\prime} \neq n} \frac{\left\langle u_{n^{\prime}, k}|\partial H(k) / \partial k| u_{n, k}\right\rangle}{\varepsilon_{n^{\prime}}(k)-\varepsilon_{n}(k)} u_{n^{\prime}}(k),
$$

where $H(k)$ is the tight binding (TB) Hamiltonian for the time independent Schrodinger equation

$$
H(k) \psi_{k}=\varepsilon_{k} \psi_{k},
$$

where $\psi_{k}$ is the wave vector containing the Bloch functions $u_{n, k(t)}$. Inserting Eq. (18) into Eq. (6) and using the orthogonality of the Bloch functions, we get

$$
X_{n, n^{\prime}}(k(t))= \begin{cases}\frac{\left\langle u_{n^{\prime}, k}|\partial H(k) / \partial k| u_{n, k}\right\rangle}{\varepsilon_{n^{\prime}}(k)-\varepsilon_{n}(k)} & n \neq n^{\prime} \\ 0 & n=n^{\prime} .\end{cases}
$$

For the case of degenerate bands at a certain $k(t)$, degenerate perturbation theory has to be used. The result of the $\mathrm{X}$ matrices obtained from using Rayleigh-Schrodinger perturbation theory is independent of the k-space gridding.

Using the finite difference method, the derivative of the Bloch function can be written as

$$
\begin{aligned}
& X_{n, n^{\prime}}(k(t)) \\
& \quad=-i \int_{\Omega} u_{n, k(t)}^{*}(x)\left(\frac{u_{n^{\prime}, k(t)+d k / 2}(x)-u_{n^{\prime}, k(t)-d k / 2}(x)}{d k}\right) d^{3} x,
\end{aligned}
$$

which can be simplified to

$$
X_{n, n^{\prime}}(k(t))=-\frac{i}{d k}\left[I_{n^{\prime} k^{+}, n, k}-I_{n^{\prime} k^{-}, n, k}\right],
$$

where

$$
I_{n^{\prime} k^{+}, n, k}=\int_{\Omega} u_{n, k}^{*}(x) \cdot u_{n^{\prime}, k^{+}}(x) \cdot d^{3} x
$$

is the overlap integral between two neighboring states, with $k^{ \pm}(t)=k(t) \pm d k / 2$ and $d k$ is a small separation in k-space. If $n=n^{\prime}$, the $\mathrm{X}$ matrix must be purely real to ensure that $\sum_{n}\left|C_{n}(t)\right|^{2}=1$. This is enforced by setting the imaginary part to be 0 in Eq. (10) when $n=n^{\prime}$; this result is similar to result obtained using Rayleigh-Schrodinger perturbation theory, where the $\mathrm{X}$ matrix value is 0 when $n=n^{\prime}$. The phase of the wave functions must be treated carefully as detailed in Ref. 17 to obtain smoothly varying $\mathrm{X}$ matrices in the complex plane across the BZ. To compare the two methods, the $\mathrm{X}$ matrices were calculated on a fine k-space grid for a $3 \mathrm{~nm}$ $\times 3 \mathrm{~nm}$ InAs nanowire along [100]. Figure 1 shows the value of the magnitude of $\mathrm{X}$ using both methods.

As can be seen in Fig. 1, the finite difference method is just as accurate as the perturbation method for a fine k-space 


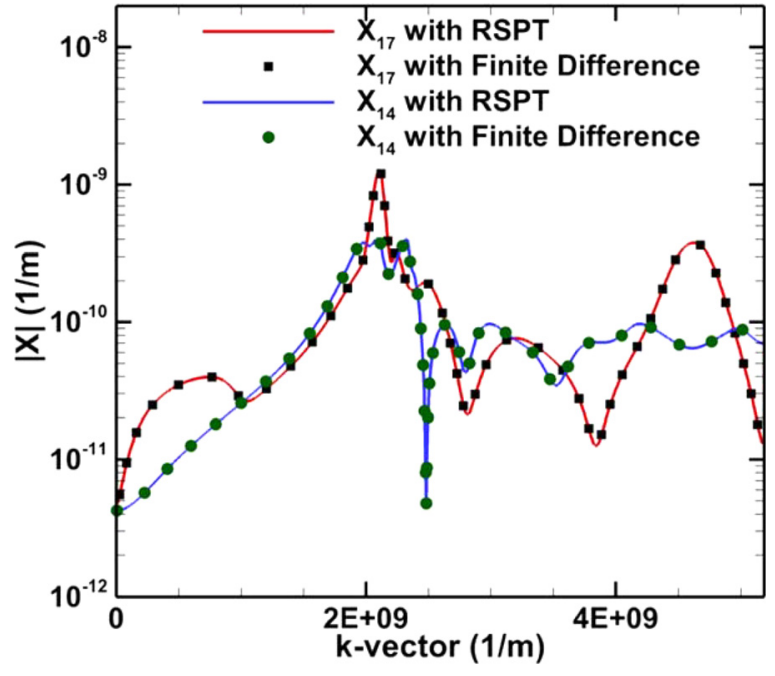

FIG. 1. The magnitude of the X matrix between band 1 and band 7, and band 1 and band 4 in a $3 \mathrm{~nm} \times 3 \mathrm{~nm}$ InAs nanowire along [100]. The symbols represent values obtained using Rayleigh-Schrodinger perturbation theory (RSPT) and the line represents values obtained using the finite difference method.

grid. The 1D BZ was equally divided into 2400 grid points on which the band structure was calculated. In all the simulations in this work, the finite difference method was used to calculate the $\mathrm{X}$ matrices.

\section{Numerical solution of the Krieger and lafrate equations in nanowires}

The band structure of the nanowire is calculated on a discrete $\mathrm{k}$-space grid using the empirical tight binding method with the $\mathrm{sp}^{3} \mathrm{~d}^{5} \mathrm{~s}^{*}$ orbitals including spin-orbit interactions. ${ }^{18}$ Since the $\mathrm{k}$-space is divided into discrete $\mathrm{k}$-cells, the solution of the probability coefficients from Eq. (12) at the $(i+1)^{t h}$ cell is given by

$$
C\left(k_{i+1}\right)=\exp \left(\Omega^{\sim}\right) C\left(k_{i}\right),
$$

where $\Omega^{\sim}$ is the truncated Magnus series expression from Eq. (13). As can be seen in Eqs. (14)-(16), the higher order terms of the Magnus series are numerically expensive to compute and involve several nested integrals. To solve them numerically, the $\mathrm{X}$ matrices are calculated at discrete $\mathrm{k}$-points, and then, a linearly interpolation scheme is used for the points in between. The energy in between the k-points is interpolated using a quadratic scheme. The individual terms of the Magnus series can then be calculated using a Gaussian quadrature of the nested integrals in Eqs. (14) and (15). The value of $\Omega^{\sim}$ up to the 4 th order in $k$ is given by ${ }^{19}$

$$
\begin{gathered}
\Omega^{\sim}=\Omega_{1}+\Omega_{2}, \\
\Omega^{\sim}=\frac{1}{2} h\left[A_{1}+A_{2}\right]-\frac{\sqrt{3}}{12} h^{2}\left[A_{1}, A_{2}\right],
\end{gathered}
$$

where $k_{i+1}=k_{i}+h$ and

$$
\begin{aligned}
& A_{1}=A\left(k_{i}+\left(\frac{1}{2}-\frac{\sqrt{3}}{6}\right) h\right) \\
& A_{2}=A\left(k_{i}+\left(\frac{1}{2}+\frac{\sqrt{3}}{6}\right) h\right),
\end{aligned}
$$

where $\left[A_{1}, A_{2}\right]$ represents the commutator operator. The value of the coefficients at the next grid point is then calculated using Eq. (24). The exponential of the matrix is calculated using the method described in Ref. 20. The 4th order Magnus (MG4) integrator described in Eq. (26) has been previously used to solve linear differential equations with a very high accuracy. ${ }^{21,22}$ Figure 2 shows the difference between using just $\Omega_{1}$ and using both $\Omega_{1}$ and $\Omega_{2}$ in Eq. (26) in solving the KI equations. Even though the difference is small, in this work, both $\Omega_{1}$ and $\Omega_{2}$ are used to solve the KI equations. It should be noted once again that even though we use just 2 terms of the Magnus series, we obtain a 4th order accurate solution in $k$.

In Ref. 22, it has been shown that the operation counts for a single time/k-vector step for a MG4 method scales as $6 n^{2}+5 n^{3}$ while the RK4 method scales as $10 n^{2}$, where $n$ is the matrix size. In our particular case, we need the probability coefficients for each permutation of initial conditions, so the RK4 method has to be resolved for every possible initial condition while the MG4 method needs to perform additional $n[n \times n][n \times 1]$ matrix multiplications (additional $n^{2}$ operations $n$ times) to obtain the final probability coefficients. The total operation counts then becomes $6 n^{2}+6 n^{3}$ for the Magnus 4th order and $10 n^{3}$ for the RK4 method for a single time/k-vector step. Therefore, for the same step size, the solutions of the KI equations using the Magnus 4th order method provides a slightly better form of solution, although they both scale as $\mathrm{O}\left(n^{3}\right)$. A limiting factor in the case of the Magnus 4th order method is the computation of the matrix exponential $\left(5 n^{3}\right)$. Nevertheless, the key difference between the RK4 method and the MG4 method lies with the choice of the time/k-vector step size. The MG4 method being inherently exponential in nature, more accurately captures the exact solution and therefore a coarser step size can be used. On the other hand, the RK4 method is a polynomial approximation and requires a much smaller step size, typically two orders of magnitude less.

Figure 3(a) shows the variation of the probability coefficient for band 1 after passing through a band crossing. The MG4 method is highly accurate for the standard step size over which the band structure is calculated. Reducing the step size by a factor of 3 does not change the result in any significant way. However, the RK4 method requires a much finer step size to obtain an accurate solution. Therefore, for a reasonably accurate solution, the RK4 method would be 100 times slower when evaluating the above band crossing. Also, the RK4 method is very unstable if the step size is below a certain size. As seen in Fig. 3(b), dividing the original step size by a factor of 1,2 , and 3 can significantly change the result and cause it to runaway exponentially if the step size is too large. This effect is problematic since there is no way of knowing the correct step size beforehand. Therefore, one has to adopt a step size and then recalculate the problem with a smaller step size till $\sum_{n}\left|C_{n}(t)\right|^{2}$ is reasonably close to 1.0. This approach is numerically expensive, and adopting the MG4 method over the RK4 method improves the computational time significantly (at least by 100 times in the above case). 


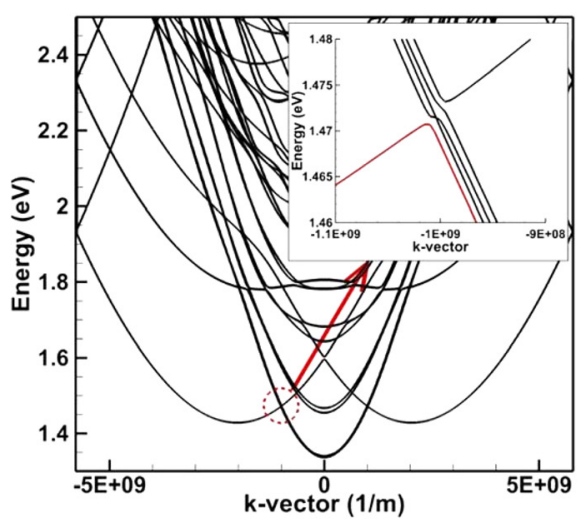

(a)

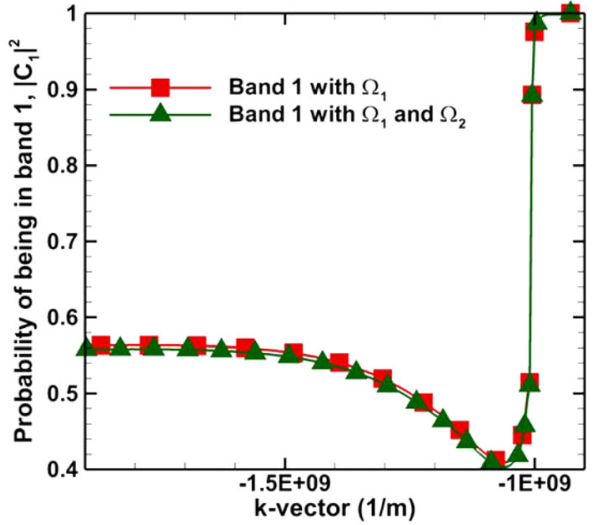

(b)

FIG. 2. (a) The conduction band of a $3 \mathrm{~nm} \times 3 \mathrm{~nm}$ Si nanowire along [100]. The inset shows a magnified section of the band structure highlighting a crossing calculated using the overlap test. ${ }^{4}$ (b) The difference between using the 1 st term and both the 1 st and 2 nd terms in the Magnus expansion on the probability coefficients for the region of the band structure shown in the inset of Fig. 2(a). The electron is initialized in the 1st band shown in red under an electric field of $10 \mathrm{kV} / \mathrm{cm}$. The square symbols represent values obtained using just the 1 st term and the delta symbols represent values obtained using both the $1 \mathrm{st}$ and 2 nd term of the Magnus expansion.

\section{Implementation of the KI equation solution within the Monte Carlo method}

As mentioned in the Introduction, the free-flight drift model of the standard Monte Carlo algorithm needs to be modified to account for interband transitions. A charge carrier undergoes a drift for a particular duration of time in the presence of a given electric field, and during this free flight period, there is a finite possibility of it transferring to the other bands. Therefore, one would ideally like to solve the KI equations for every carrier during its free-flight, at its current position in $\mathrm{k}$-space, and calculate the probability of undergoing a transition to the other bands. However, this is impractical as there are typically anywhere between $10^{7}$ and $10^{11}$ carrier drifts in a traditional Monte Carlo routine, and a significant increase in the computational time for each carrier free flight greatly affects the overall simulation run time. A less computationally expensive approach taken here is to create a lookup table, storing the transition probabilities for every possible initial condition and for a finite number of points in k-space. During runtime, depending on the carrier's initial band and the position in k-space, the corresponding transition probability table is pulled up and a random number is used to decide the final band of the carrier after the end of the free flight. The memory required to store the transition probability tables is negligible compared to the already existing cost of storing the scattering tables within the full band Cellular Monte Carlo (CMC) scheme. ${ }^{2}$ The algorithm described above, and the results presented in Section III, apply to the case of Monte Carlo simulations under a uniform electric field (so-called k-space simulation). However, the look-up table approach can be generalized to different fields as well, with the size of the look-up table still much less than the scattering tables used in the CMC algorithm. It is important to mention here that if the electric field varies significantly across the device some consideration has to be taken before using the KI equations. A key assumption in the derivation of the KI equations is treating the effect of the field in terms of a vector potential instead of a scalar potential (using the Weyl gauge). The scalar potential contribution cannot be ignored if the field has a significant spatial gradient and the full Lorentz gauge has to be used. In situations where the inhomogeneity of the field is localized and strong as it might be in the case of impurities, heterojunctions, or quantum wells, the wave functions have to be represented in terms of localized basis states as the difficulty mainly arises if the nature of the basis states is extended as discussed in

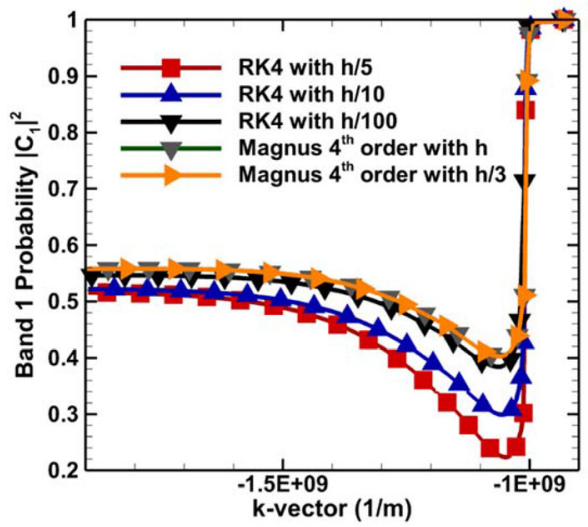

(a)

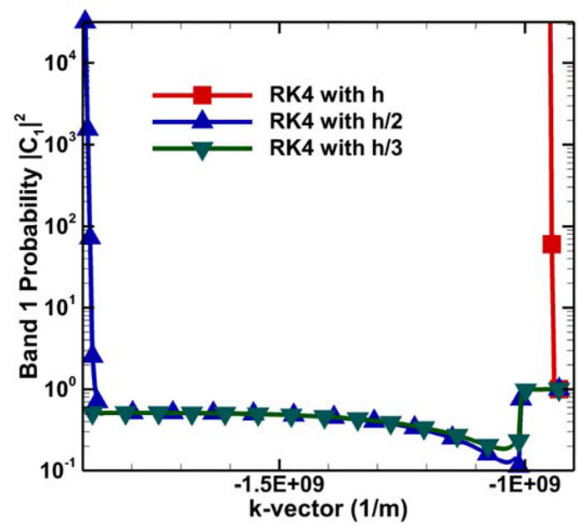

(b)
FIG. 3. (a) The difference between using different step sizes for the Magnus 4th order and the Runge-Kutta 4 th order (RK4) method. The region of the band structure simulated is shown in Fig. 2(a) inset. The band 1 is highlighted in red. (b) The sensitivity of the RK4 method to coarse step sizes is shown. $h$ is the step size on which the band structure is calculated. 
Ref. 23. In these cases, it is advantageous to use the TB method where the basis states are localized as opposed to the EPM method where the basis states are extended.

To implement this method, a step size $\Delta k$ for the $\mathrm{k}$-space grid is required. The grid on which the band structure is calculated is usually too coarse. To identify the correct $\Delta k$, a minimum electric field $F_{\min }$ is used. Below $F_{\min }$, the effect of multi-band drift is assumed to be unimportant, and $\Delta k$ can then be calculated as

$$
\Delta k=\frac{e F_{\min } t_{d r i f t}}{\hbar} .
$$

The 1D BZ is then divided equally into $N$ k-points separated by $\Delta k$, where $N$ is given by

$$
N=\frac{2 \pi}{a \Delta k},
$$

where $a$ is the periodicity of the supercell along the nanowire axis. The KI equations are solved for the duration of the drift and the transition probabilities for every k-point and initial band index are then stored for a particular electric field $F$ and drift time, $t_{\text {drift }}$. The electric fields used in the uniform field simulation are then chosen to be integer multiples of $F_{\min }$. This is important so as to correctly capture the transition probabilities. If $F / F_{\min }=p$, where $p$ is not an integer, then after a drift time $t_{d r i f t}$, the final $\mathrm{k}$-value of the carrier will be

$$
k_{f}=k_{i}+p \Delta k,
$$

where $k_{i}$ is the initial $\mathrm{k}$-value. Since the transition probabilities are only stored for every $\Delta k$, there will be an error in the stored transition probabilities during the actual carrier drift. There still exists an error after a carrier undergoes a scattering event, as its momentum will in general not lie on the k-points of the pre-calculated transition tables. To minimize this error, $F_{\text {min }}$ must be chosen as small as computationally possible. In this work, $F_{\min }$ was chosen to be $2 \times 10^{5} \mathrm{~V} / \mathrm{m}$, which gives a $\Delta k$ of $1.22 \times 10^{5} \mathrm{~m}^{-1}$ for a drift time of $4 \times 10^{-16} \mathrm{~s}$. This gives a total of $N=94843 \mathrm{k}$-points in the finer k-space grid for a Si nanowire along [100]. The exponential in Eq. (24) is the transition probability from $k_{i}$ to $k_{i+1}$. Depending on the electric field and drift time, the final transition probability matrix is calculated by successively multiplying the matrices for the required number of k-cells obtained using Eq. (30)

$$
C\left(k_{f}\right)=T_{f} \ldots . . T_{i+2} T_{i+1} T_{i} C\left(k_{i}\right) .
$$

Once the final transition matrix from $k_{i}$ to $k_{f}$ is calculated, the probability coefficients for every possible initial condition is calculated and stored.

In the present work, the $\mathrm{Si}$ and InAs nanowire band structures are calculated using the semi-empirical $s p^{3} d^{5} s^{*}$ Tight Binding (TB) model including the spin-orbit interaction. ${ }^{18}$ Deformation potential scattering rates are calculated from the TB coefficients using the method outlined in Ref. 24. The polar optical phonon scattering rates are calculated by the method described in Ref. 25. Impact ionization is not included in this work. The carriers (electrons and holes) are initialized according to a one-dimensional Maxwell distribution at room temperature. The traditional full band Monte Carlo approach is then performed with the inclusion of the modified free-flight drift routine to account for the interband transitions after the free flight.

\section{RESULTS AND DISCUSSION}

The usefulness of the KI equations is the ability to simulate a field dependent probability of interband transitions. In Fig. 4(a), a section of $3 \mathrm{~nm} \times 3 \mathrm{~nm}$ InAs nanowire band structure along [100] is magnified to demonstrate interband tunneling. The electron is initially in band 1 , shown in red in the inset of Fig. 4(a) and undergoes drift for different constant electric fields. The initial k-point is $-3.3 \times 10^{9} \mathrm{~m}^{-1}$ and the final k-point is $-4.4 \times 10^{9} \mathrm{~m}^{-1}$. The probability of the electron being in various bands is presented as $|C|^{2}$ and is shown in Figs. 4(b), 4(c), and 4(d) for electric fields of 10 $\mathrm{kV} / \mathrm{cm}, 100 \mathrm{kV} / \mathrm{cm}$, and $1 \mathrm{MV} / \mathrm{cm}$, respectively. In all plots of band structures, the band indices at different $\mathrm{k}$ points are determined using the overlap test. This is done so as to easily talk about carriers moving across the BZ and has no significance on the physics of the interband tunneling process.

At $10 \mathrm{kV} / \mathrm{cm}$, the probability of an electron remaining in band 1 is close to unity since the electric field is not high enough to induce interband transitions as can be seen in Fig. 4(b) for the energy separation between the bands shown in Fig. 4(a). As can be seen in Figs. 4(c) and 4(d), at higher electric fields of $100 \mathrm{kV} / \mathrm{cm}$ and $1 \mathrm{MV} / \mathrm{cm}$, the electron tunnels to the 2nd and 3rd band with increasingly higher probability with field, with the highest probability being to the 3rd band even though the energy separation between the 1st and $3 \mathrm{rd}$ band is higher than that between the 1st and 2nd bands. This somewhat non-intuitive result is due to the fact that the $\mathrm{X}$ matrices are higher between the 1st and 3rd band as compared to the 1st and 2nd band due to the overlap of the wave functions related to symmetry.

A similar case is shown for a $3 \mathrm{~nm} \times 3 \mathrm{~nm}$ Si nanowire along [100] in Fig. 5(a), where we have the case of an actual band crossing/anti-crossing. The initial k-point is -9.0 $\times 10^{8} \mathrm{~m}^{-1}$ and the final k-point is $-1.9 \times 10^{9} \mathrm{~m}^{-1}$. The band crossing/anti-crossing occurs at $-1.0 \times 10^{9} \mathrm{~m}^{-1}$. Due to the very low energy separation near the band crossing, interband tunneling occurs even at electric fields as low as $1.0 \mathrm{kV} / \mathrm{cm}$, as shown in Fig. 5(b). As the carrier drifts across the band crossing, the probability that the electron remains in the 1 st band reduces drastically. The probability of transition is independent of the electric field in this case since the energy bands are very close to one other. In Eq. (11) when the energy difference between bands goes to 0 , the KI equations become independent of the electric field as is evident in Figs. 5(b) and 5(c). Therefore, interband tunneling is very important and requires an accurate solution of the KI equations across the full BZ.

In Figs. 6 and 7, the effect of the multi-band drift model on the average kinetic energy of the carriers from a uniform field Cellular Monte Carlo simulation is shown.

In Figs. 6(a) and 6(b), the conduction and valence band of $3 \mathrm{~nm} \times 3 \mathrm{~nm}$ Si nanowire along the [111] direction is 


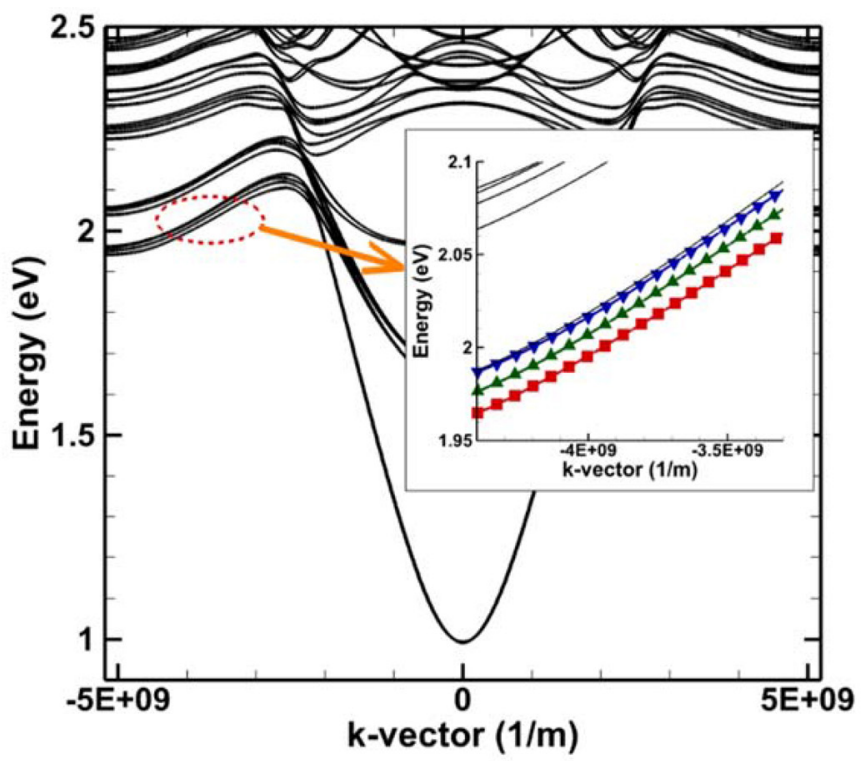

(a)

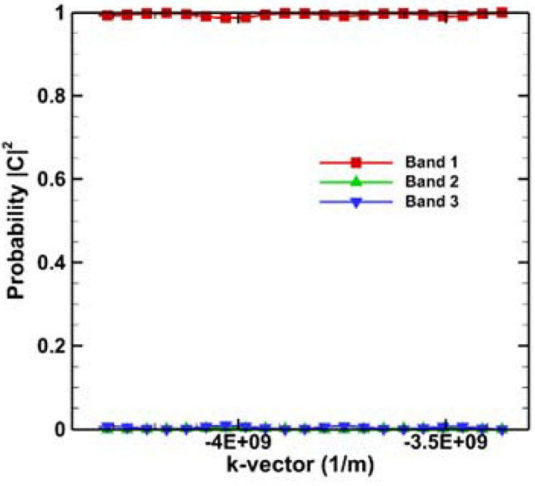

(b)

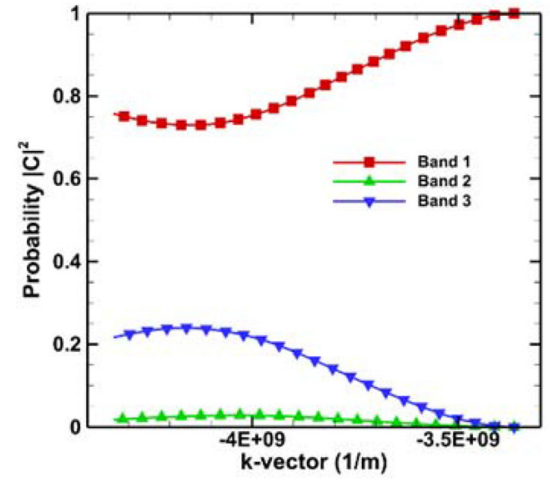

(c)

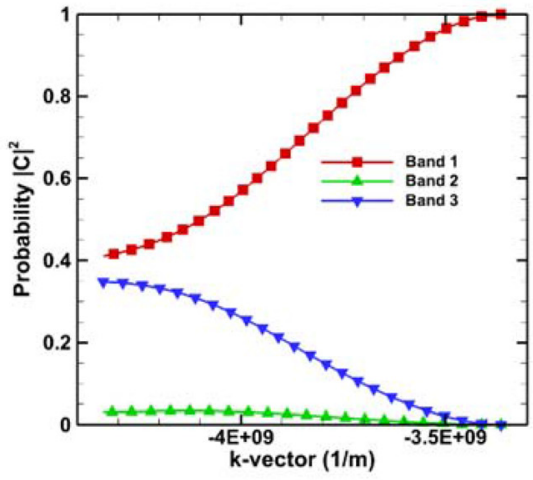

(d)

FIG. 4. (a) Band structure of a $3 \mathrm{~nm} \times 3 \mathrm{~nm}$ InAs nanowire band structure along [100]. The inset shows a magnified part of the band structure showing bands close to one other. The 1st band is represented by square symbols, the 2 nd band by left triangles and the 3rd band by right triangles. (b) Plot of transition probabilities as a function of $k(t)$ under an applied electric field of (b) $10 \mathrm{kV} / \mathrm{cm}$, (c) $100 \mathrm{kV} / \mathrm{cm}$, and (d) $1 \mathrm{MV} / \mathrm{cm}$ for the first three bands at the location shown in the inset of Fig. 3(a).

shown. In the case of the $3 \mathrm{~nm} \times 3 \mathrm{~nm}$ Si nanowire along the [111] direction, in the valence band shown in Fig. 6(b), there exists a number of band anti-crossings; therefore, the carriers are unable to reach high energies with the traditional drift algorithm and the addition of the multi-band drift significantly increases the average energy. Similarly in Fig. 6(a), without the multi-band drift approach, the carriers' energy saturates as carriers are unable to reach high enough energies with just inelastic scattering, as can be seen in Figs. 6(c) and $6(\mathrm{~d})$. In the case of $3 \mathrm{~nm} \times 3 \mathrm{~nm}$ Si nanowires, the multiband drift model makes more of a difference for [111] $\mathrm{Si}$ compared to [100] Si for both electrons and holes, due to the differences in band structure between the two in the direction of the electric field.

The effect of the multi-band drift is very apparent in the case of the electrons in $3 \mathrm{~nm} \times 3 \mathrm{~nm}$ InAs nanowires. In Figs. 7(a) and 7(b), the conduction band of $3 \mathrm{~nm} \times 3 \mathrm{~nm}$ InAs nanowires along the [100] and [111] directions are shown. In the [100] direction bands, there are several anticrossings near $2.2 \mathrm{eV}$ (K.E. $\sim 1.2 \mathrm{eV}$ ) which causes the energy to saturate in the absence of interband tunneling. The same situation is present in the [111] direction bands as there is a small band gap present between the first two conduction bands, and the rest of the conduction bands. This gap would only be crossable with inelastic scattering processes such as polar and non-polar optical phonon scattering in a traditional Monte Carlo. This effect becomes apparent with consideration of the average energy of the electrons, which saturate at $0.6 \mathrm{eV}$ above the conduction band minima as seen in Fig. 7(c). When the multi-band drift model is employed, the electrons achieve much higher energies due to interband tunneling. The average kinetic energy of the holes is plotted in Fig. 7(d). The average kinetic energies of the holes when multiband drift model is used are higher than the case when it is not used, although the change in energy is not as high as it is in the case of electrons.

Figures 8(a)-8(d) demonstrate the difference in carrier populations with and without the inclusion of multi-band transport. In Fig. 8(a), a snapshot of 10000 electrons is shown for a $3 \mathrm{~nm} \times 3 \mathrm{~nm}$ InAs nanowire along the [111] direction at an electric field of $1 \mathrm{MV} / \mathrm{cm}$ for the case of a conventional CMC simulation, where the electrons are 


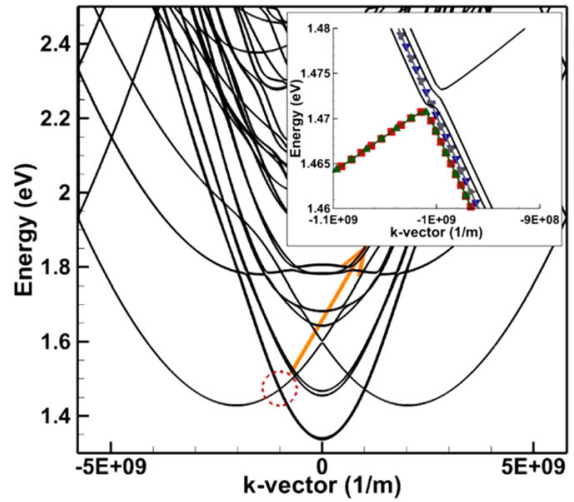

(a)

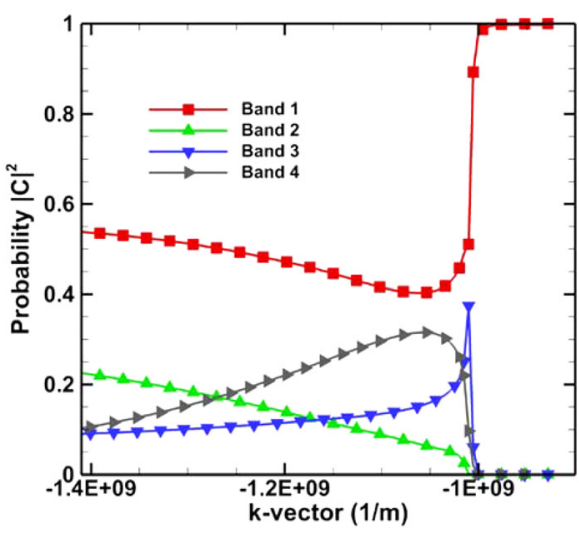

(b)

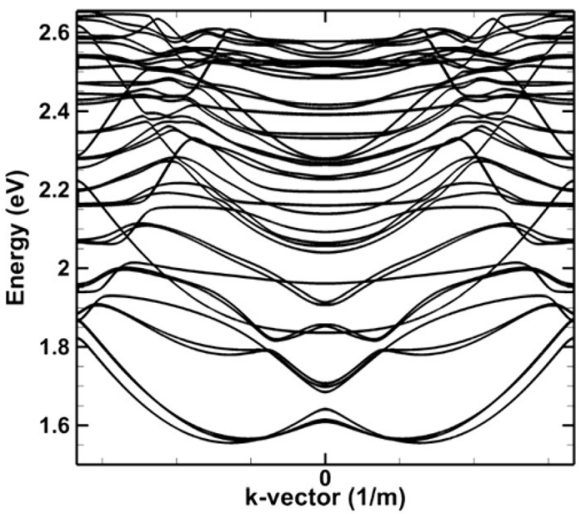

(a)

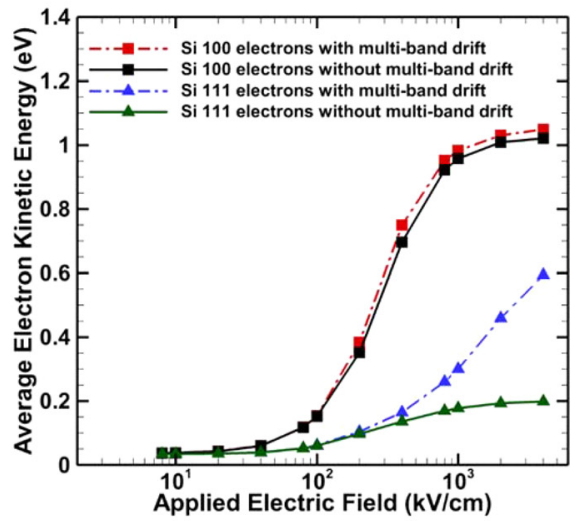

(c)

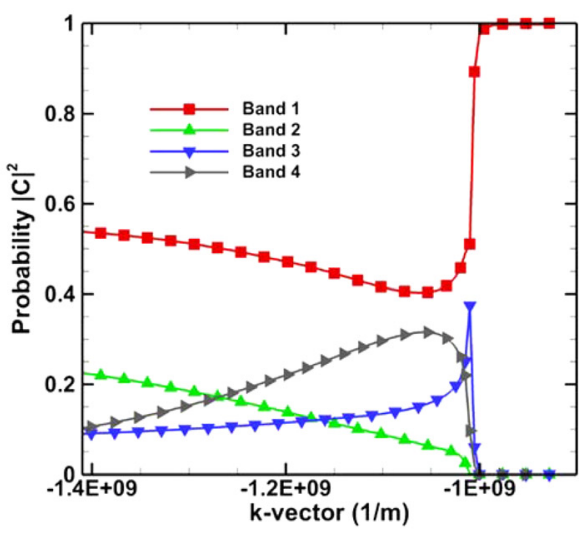

(c)

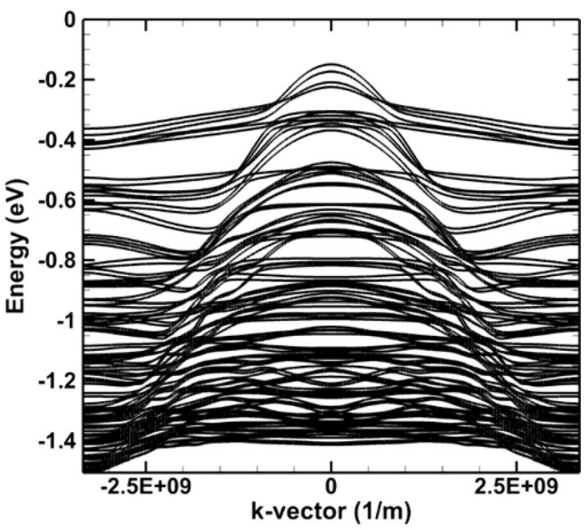

(b)

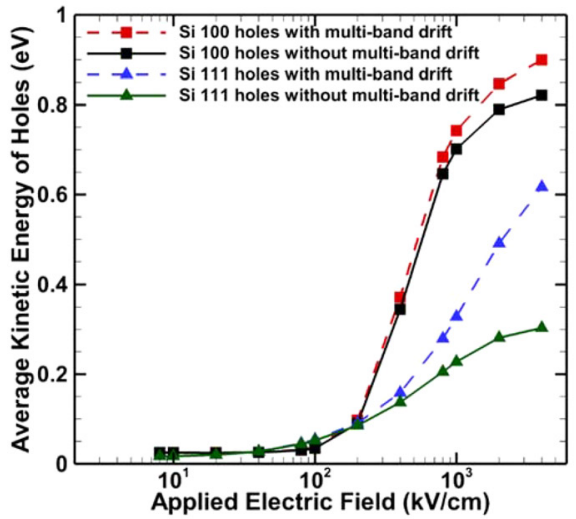

(d)
FIG. 5. (a) Band structure of a $3 \mathrm{~nm}$ $\times 3 \mathrm{~nm}$ Si nanowire along the [100] direction. The inset shows a magnified part of the band structure showing a band crossing. The first four bands are colored and marked. (b) Plot of transition probabilities as a function of $k(t)$ under an applied electric field of (b) $1.0 \mathrm{kV} / \mathrm{cm}$ and (c) $100 \mathrm{kV} / \mathrm{cm}$ for the first four bands at the band crossing shown in the inset of (a).
FIG. 6. (a) Conduction band structure of $3 \mathrm{~nm} \times 3 \mathrm{~nm}$ Si nanowire along the [111] direction. (b) Valence band structure of $3 \mathrm{~nm} \times 3 \mathrm{~nm}$ Si nanowire along the [111] direction. (c) Plot of average electron kinetic energies in $3 \mathrm{~nm} \times 3 \mathrm{~nm}$ Si nanowires along the [100] and [111] directions with and without multi-band drift. (d) Plot of average hole kinetic energies in $3 \mathrm{~nm}$ $\times 3 \mathrm{~nm}$ Si nanowires along the [100] and [111] directions with and without multi-band drift. 


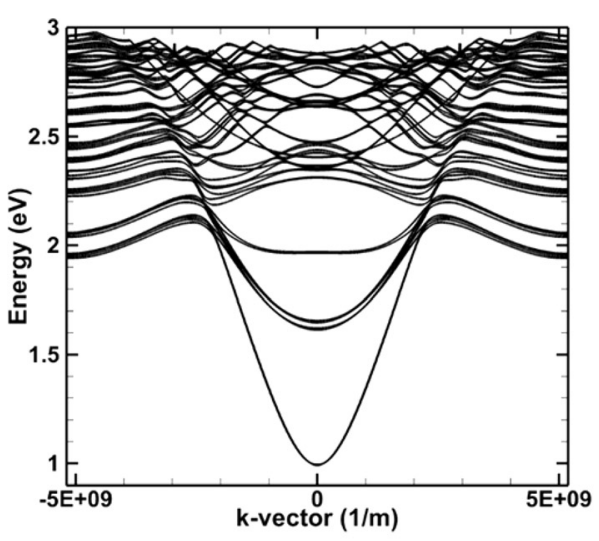

(a)

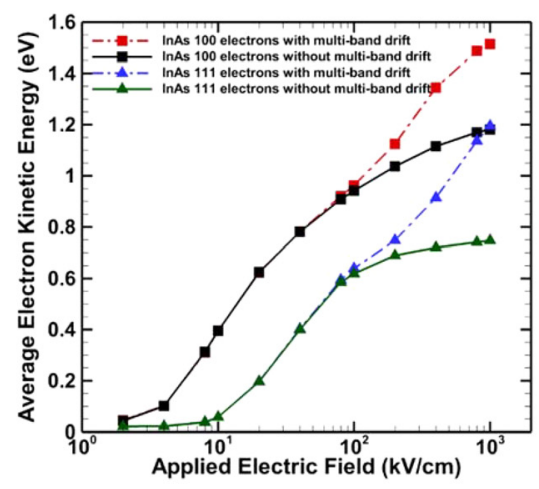

(c)

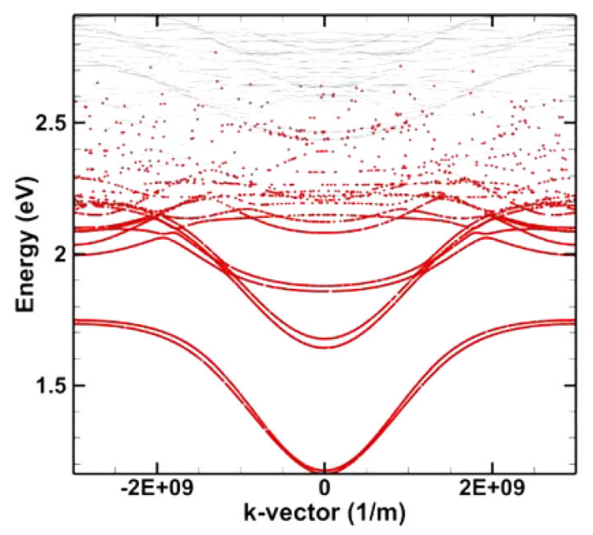

(a)

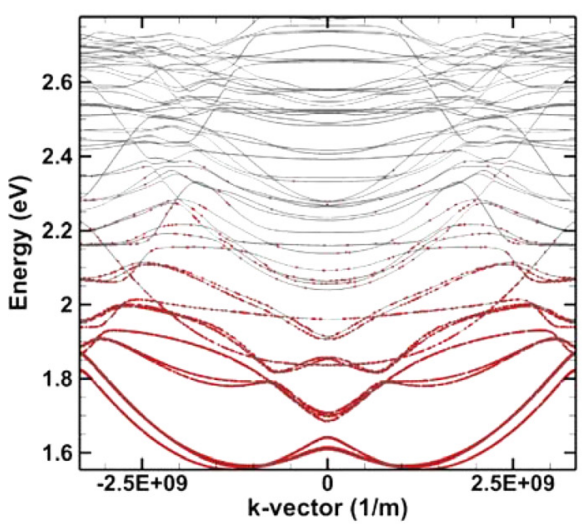

(c)

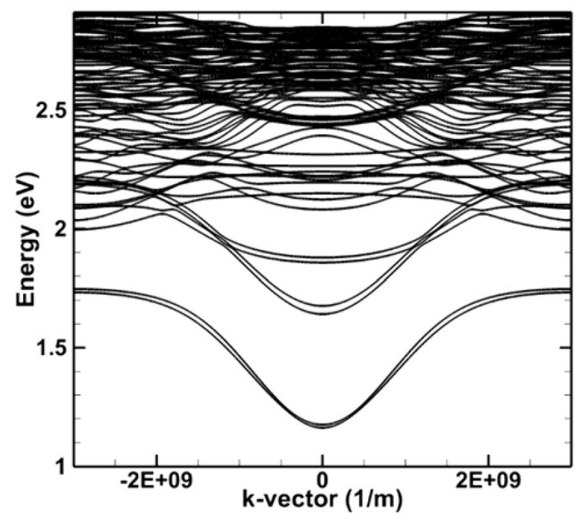

(b)

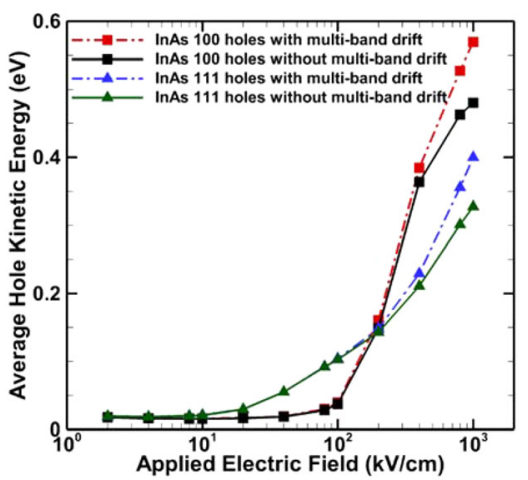

(d)

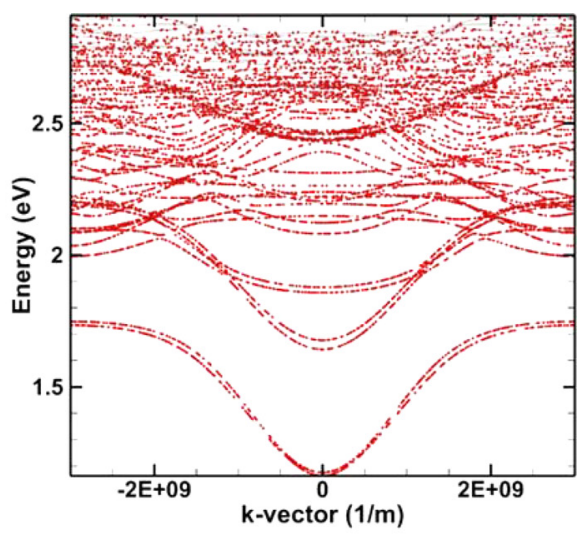

(b)

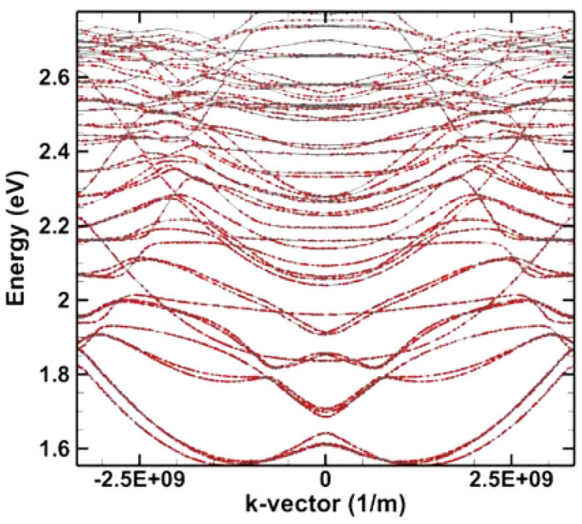

(d)
FIG. 7. (a) Conduction band structure of $3 \mathrm{~nm} \times 3 \mathrm{~nm}$ InAs nanowire along [100]. (b) Conduction band structure of $3 \mathrm{~nm} \times 3 \mathrm{~nm}$ InAs nanowire along [111]. (c) Plot of average electron kinetic energies in $3 \mathrm{~nm} \times 3 \mathrm{~nm}$ InAs nanowires along [100] and [111] with and without multi-band drift. (d) Plot of average hole kinetic energies in $3 \mathrm{~nm} \times 3 \mathrm{~nm}$ InAs nanowires along [100] and [111] with and without multi-band drift.
FIG. 8. (a) Snapshot of electrons in the conduction band of $3 \mathrm{~nm} \times 3 \mathrm{~nm}$ InAs along [111] at the end of the simulation without multi-band drift at $1 \mathrm{MV} / \mathrm{cm}$. Each red dot represents an electron. (b) Snapshot of electrons in the conduction band of $3 \mathrm{~nm} \times 3 \mathrm{~nm}$ InAs along [111] at the end of the simulation with multiband drift at $1 \mathrm{MV} / \mathrm{cm}$. (c) Snapshot of electrons in the conduction band of $3 \mathrm{~nm} \times 3 \mathrm{~nm} \mathrm{Si}$ along [111] at the end of the simulation without multi-band drift at $4 \mathrm{MV} / \mathrm{cm}$. Each red dot represents an electron. (d) Snapshot of electrons in the conduction band of $3 \mathrm{~nm}$ $\times 3 \mathrm{~nm} \mathrm{Si}$ along [111] at the end of the simulation with multi-band drift at 4 $\mathrm{MV} / \mathrm{cm}$. 
unable to reach higher bands. When the multi-band drift algorithm is employed, carriers are able to access much high energies as is shown in Fig. 8(b). A similar case for $\mathrm{Si}$ is shown in Figs. 8(c) and 8(d) at an electric field of $4 \mathrm{MV} / \mathrm{cm}$.

\section{CONCLUSION}

In the present paper, we presented a new solution of the KI equations for multi-band transport using the Magnus expansion method. The usefulness of the Magnus expansion to solve the problem of multi-band drift is demonstrated by using it to solve the KI equation across the full BZ in semiconductor nanowire systems. The ability of the Magnus solution to retain qualitative properties of the original solution greatly simplifies the problem. Depending on the problem at hand, the Magnus series can also be accordingly truncated to the required degree of accuracy, reducing the computation time without introducing exponentially increasing errors as is the case with the Runge-Kutta 4th order method. The importance of field induced interband tunneling is shown by performing uniform field full band Monte Carlo simulations of $\mathrm{Si}$ and InAs nanowires by modifying the traditional freeflight drift routine. Depending on the nature of the band structure, the multi-band drift model may or may not be necessary. Interband tunneling also becomes very important at high electric fields where impact ionization may be dominant. In such cases, it is important to include this model in the traditional Monte Carlo routines to accurately account for the correct number of impact events at high electric fields.

\section{ACKNOWLEDGMENTS}

The authors would like to thank the Numerical Device Modeling group at Intel, OR, for support of this research.
${ }^{1}$ M. Feraille, D. Rideau, A. Ghetti, and A. Poncet, in 2006 International Conference on Simulation of Semiconductor Processes and Devices (2006), pp. 264-266.

${ }^{2}$ M. Saraniti and S. M. Goodnick, IEEE Trans. Electron. Devices 47, 1909 (2000).

${ }^{3}$ R. Hathwar, M. Saraniti, and S. M. Goodnick, J. Phys.: Conf. Ser. 647, 012029 (2015).

${ }^{4}$ C. Jacoboni and L. Reggiani, Rev. Mod. Phys. 55, 645 (1983).

${ }^{5}$ M. Fischetti, IEEE Trans. Electron Devices 38, 634-649 (1991).

${ }^{6}$ H.-E. Nilsson, A. Martinez, E. Ghillino, U. Sannemo, E. Bellotti, and M. Goano, J. Appl. Phys. 90, 2847 (2001).

${ }^{7}$ E. Bellotti, H.-E. Nilsson, K. F. Brennan, P. P. Ruden, and R. J. Trew, J. Appl. Phys. 87, 3864 (2000).

${ }^{8}$ K. F. Brennan, E. Bellotti, M. Farahmand, H.-E. Nilsson, P. P. Ruden, and Y. Zhang, IEEE Trans. Electron Devices 47, 1882 (2000).

${ }^{9}$ J. B. Krieger and G. J. Iafrate, Phys. Rev. B 33, 5494-5500 (1986).

${ }^{10}$ F. Bertazzi, M. Moresco, and E. Bellotti, J. Appl. Phys. 106, 063718 (2009).

${ }^{11}$ W. Magnus, Commun. Pure Appl. Math. 7(4), 649-673 (1954).

${ }^{12}$ S. Blanes, F. Casas, J. A. Oteo, and J. Ros, Phys. Rep. 470, 151-238 (2009).

${ }^{13}$ A. Iserles and S. P. Nørsett, Philos. Trans. R. Soc. London, Ser. A 357, 983-1019 (1999).

${ }^{14}$ F. Fer, Acad. Roy. Belg. Bull. Cl. Sci. 44, 818-829 (1958).

${ }^{15}$ R. M. Wilcox, J. Math. Phys. 8, 962 (1967).

${ }^{16}$ E. Schrödinger, Ann. Phys. 385, 437-490 (1926).

${ }^{17}$ U. Lindefeldt, H.-E. Nilsson, and M. Hjelm, Semicond. Sci. Technol. 19, 1061-1066 (2004).

${ }^{18}$ M. Luisier, A. Schenk, W. Fichtner, and G. Klimeck, Phys. Rev. B 74, 205323 (2006).

${ }^{19}$ A. Iserles, A. Marthinsen, and S. P. Nørsett, BIT Numer. Math. 39, 281-304 (1999).

${ }^{20}$ C. Moler and C. VanLoan, SIAM Rev. 45(1), 3-49 (2003).

${ }^{21}$ L. Wen-cheng, D. Zi-chen, and H. Yong-an, Appl. Math. Mech. 27, 1383-1390 (2006).

${ }^{22}$ N. D. Aparicio, S. J. A. Malham, and M. Oliver, BIT Numer. Math. 45(2), 219-258 (2005).

${ }^{23}$ G. J. Iafrate and J. B. Krieger, Phys. Rev. B 40, 9 (1989).

${ }^{24}$ A. K. Buin, A. Verma, and M. P. Anantram, J. Appl. Phys. 104, 053716 (2008).

${ }^{25}$ R. Hathwar, M. Saraniti, and S. M. Goodnick, in 2014 IEEE 14th International Conference on Nanotechnology (2014), pp. 645-649. 